\title{
Chemotherapy in metastatic malignant triton tumor: Report on two cases
}

\author{
N.H. THOENNISSEN ${ }^{1}$, C. SCHLIEMANN ${ }^{1}$, U. BRUNNBERG ${ }^{1}$, E. SCHMIDT ${ }^{1}$, A. STAEBLER ${ }^{2}$, L. STEGGER ${ }^{3}$, \\ C. BREMER ${ }^{4}$, C. SCHLEICHER ${ }^{5}$, R.M. MESTERS ${ }^{1}$, C. MÜLLER-TIDOW ${ }^{1}$ and W.E. BERDEL ${ }^{1}$
}

\author{
Departments of ${ }^{1}$ Medicine, Hematology and Oncology, ${ }^{2}$ Pathology, ${ }^{3}$ Nuclear Medicine, \\ ${ }^{4}$ Radiology and ${ }^{5}$ Surgery, University of Münster, D-48149 Münster, Germany
}

Received April 10, 2007; Accepted June 28, 2007

\begin{abstract}
Malignant triton tumor (MTT) is a rare, highly malignant nerve sheath tumor with rhabdomyoblastic differentiation. Initial debulking surgery followed by adjuvant therapy is the current treatment of choice, but has very limited efficacy when optimal cytoreduction is not achieved by surgical procedure. Neoadjuvant therapy for MTT, to potentially facilitate subsequent surgery, eradicate micrometastatic lesions and, therefore, improve the therapeutical outcome, has never before been presented in literature. Here, we report on the multimodal management of two cases of advanced and metastatic MTT. Treatment modalities involved neoadjuvant and adjuvant chemotherapy, surgical resection, and radiation. In both cases, integrated Positron Emission Tomography/Computed Tomography (PET/CT) emerged as an important diagnostic tool for the reliable assessment of MTT response and metabolic remission.
\end{abstract}

\section{Introduction}

Malignant triton tumor (MTT) is a very rare and highly aggressive variant of the malignant peripheral nerve sheath tumor (MPNST), with rhabdomyoblastic differentiation. MPNSTs as a group account for approximately $5 \%$ of all soft tissue sarcomas (STS) (1). MTT usually develops in individuals younger than 35 and is associated with a very poor outcome; crude 2- and 5-year survival rates are 15 and $11 \%$, respectively (2-4). In literature reviews, approximately half to two thirds of cases occur in the context of neurofibromatosis type 1 (NF-1) (5), but this is no 'conditio sine qua non' for the appearance of MTT. Because of its rarity, treatment strategies - especially in advanced and metastatic disease - are

Correspondence to: Dr N.H. Thoennissen, Department of Medicine, Hematology and Oncology, University of Münster, Albert Schweitzer Str. 33, D-48149 Münster, Germany

E-mail: n.thoennissen@gmx.de

Key words: malignant triton tumor, chemotherapy, positron emission tomography not well established. The main therapeutic strategy for MTT, as reported, is identical to that of most STSs and often requires multimodal therapy. Total resection of the tumor is the most important therapeutical goal. When radical tumor removal is not possible, excision combined with high-dose radiation therapy is thought to be an alternative treatment (6). Although postoperative chemotherapy could be a part of multimodal therapy, its therapeutic value is not proven $(1,7)$. Neoadjuvant therapy in MTT, in a multimodal setting which has never been described before, could be a new and important module to yield tumor response.

Here, we present two cases of MTT which were diagnosed, evaluated and treated at our medical centre. The first report describes a 25-year-old woman, without NF-1, suffering from advanced MTT with hepatic, iliocecal and parauterine manifestations. To the best of our knowledge, this is the first report to be published on the management of advanced metastatic MTT by neoadjuvant chemotherapy followed by surgical resection and adjuvant chemotherapy. The second case concerns a 24-year-old man, with known NF-1, given the initial diagnosis of localized MTT with cervical manifestation. He was initially treated with surgery followed by high-dose radiation therapy. Later on, he was treated with chemotherapy because of early progressive disease with pulmonary metastasis. Although Magnetic Resonance Imaging (MRI) has been described as the imaging modality of choice for MTT $(1,8)$, in both cases we successfully used additional Positron Emission Tomography/Computed Tomography (PET/CT) as a helpful tool to assess tumor regression and metastatic extent in the therapeutic management.

\section{Case reports}

Case 1. A 25-year-old woman was admitted to local hospital after a 2-week history of right upper abdominal pain radiating into the right shoulder. Her medical history revealed surgical resection, 3 months before, of a Sertoli-cell-tumor localized to the left ovary (left adnectomy, lymphonodectomy, omentectomy), which was diagnosed following a missed abortion. The family history and the clinical examination were negative for NF-1.

In further evaluation, a CT scan of the neck, chest and abdomen presented a large heterogeneous hepatic mass $(8 \times 12 \mathrm{~cm}$ in diameter) and a smaller left parauterine mass 


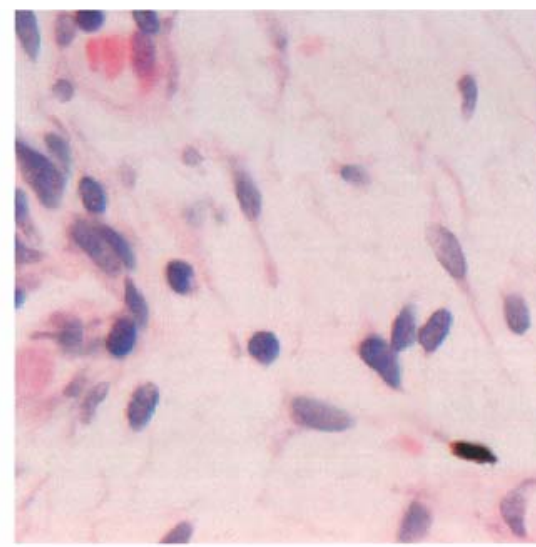

A

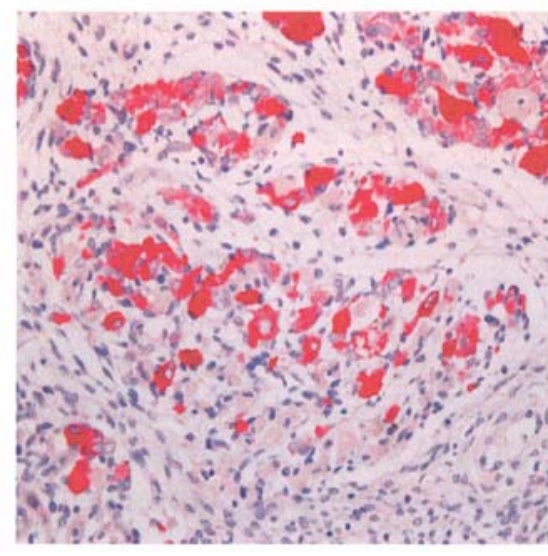

C

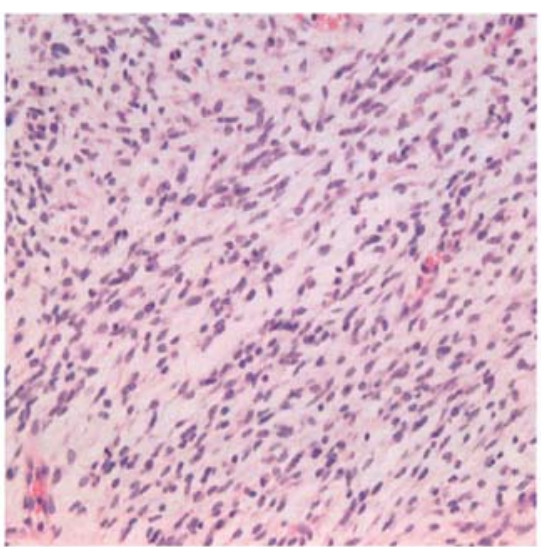

B

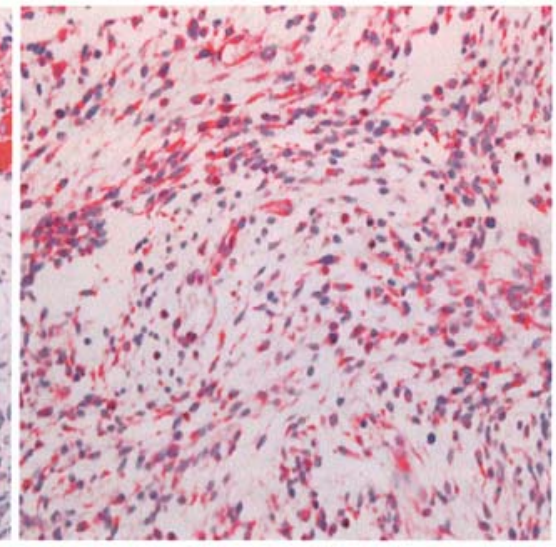

D

Figure 1. (A) Large, pleomorphic rhabdoid cells with abundant eosinophilic cytoplasm are embedded in a spindle cell tumor with fine fibrillary matrix. (B) Spindle cell tumor with a fine fibrillary matrix and numerous mitotic figures. (C) Immunohistochemical stain of desmin, strong and specific staining in the rhabdoid tumor cells indicating the rhabdomyosarcomatous component of the tumor. (D) Immunohistochemical stain for neuron-specific-enolase (NSE), strong and diffuse staining in the spindle cells indicating the neurogenic component of the tumor.

$(4.5 \times 5 \mathrm{~cm})$. Early tumor relapse of the known Sertoli-celltumor was highly suspected. Surgical removal of the parauterine tumor through hysterectomy and a right adnectomy was carried out. On macroscopic inspection, the tumor was gray-brown, elastic and attached to the uterus with infiltration of the uterine serosa. Complete surgical resection of the parauterine tumor was performed with all margins negative for residual tumor tissue (R0). On further histopathological examination, the neoplastic tissue displayed a high cellularity with large areas of spindle-shaped tumor cells, and scattered, round cells with wide eosinophilic cytoplasm and atypical nuclei, identified as rhabdomyoblasts (Fig. 1A). Mitotic figures were frequently seen (Fig. 1B) and immunohistochemistry demonstrated strong positive staining of the rhabdomyoblastic cells for desmin and variable staining for sarcomeric actin (Fig. 1C). Nerve sheath differentiation of the spindle cells was confirmed by S-100 and vimentin protein positivity (Fig. 1D). Immunostainings using an antibody against cytokeratin were negative, excluding an epithelial origin of the neoplastic tissue. Molecular characterization showed no mutation of c-Kit (CD117). In conclusion, the biphenotypic appearance of the tumor led to the unanticipated diagnosis of MTT $(9,10)$. The initial diagnosis of Sertoli-cell- tumor in the patient's past medical history was also confirmed by a re-evaluation of the extracted left ovary by an independent reference pathologist. In addition, histological examination of a CT-guided biopsy of the hepatic mass showed MTT tissue and underlined the unexpected diagnosis of an advanced stage of the rare tumor entity.

The patient was transferred to our department and we performed a whole body FDG-PET/CT-scan (Fig. 2B) for further staging. It demonstrated three abdominal tumor manifestations. The largest one, seen before in the CT scan, was now taking over the complete right lobe of the liver (26x16x17 cm, SUV 10.8; Fig. 2A and B), the appearance was heterogeneous with central liquid. Remarkably, the hepatic mass had progressed from $8 \times 12$ to $16 \times 26 \mathrm{~cm}$ within only a month, in keeping with the highly aggressive character of MTT. Additionally, two new manifestations were found in the right lower quadrant of the abdomen next to the ileocecum $(3 \times 3.5 \mathrm{~cm}$, SUV 8.7) and cranial to the urinary bladder (1.7x2.8 cm, SUV 6.5; Fig. 2B). The hepatic mass was deemed unresectable due to its large size.

To potentially enhance the tumor's resectability, the patient received three systemic neoadjuvant cycles of PEI chemotherapy, consisting of cisplatin 20, etoposide 75 and ifosfamide 


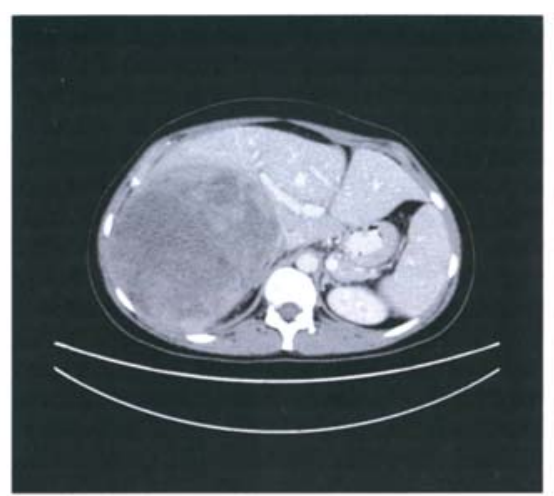

A
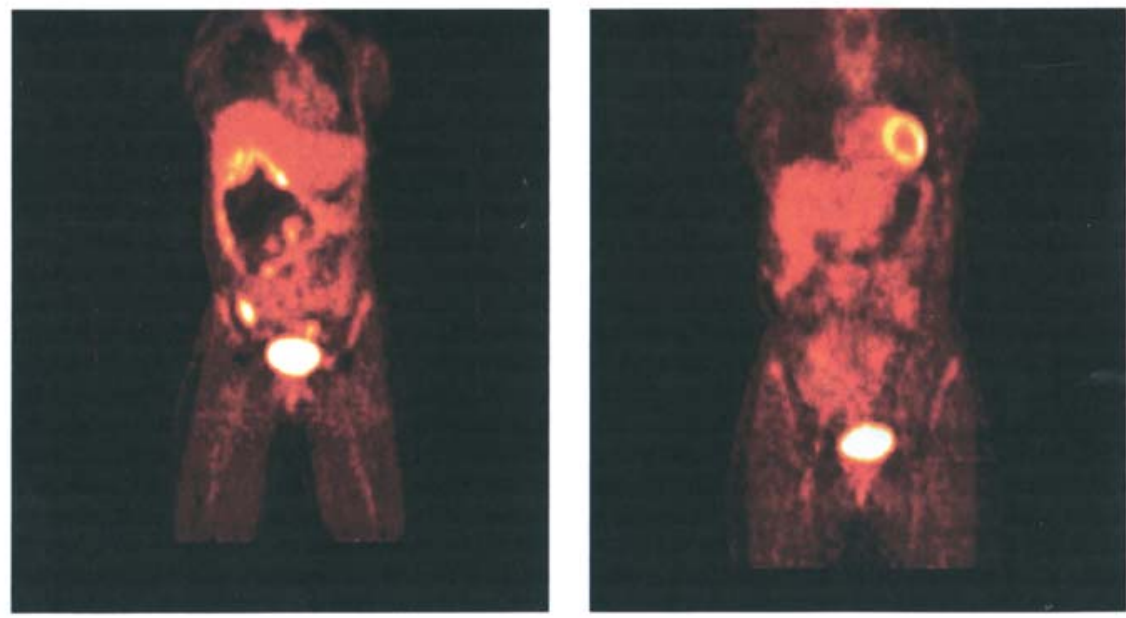

B

C

Figure 2. (A) CT abdomen, status before treatment with MTT of the liver $(26 \times 16 \times 17 \mathrm{~cm})$ taking over the complete right lobe, the appearance was heterogeneous with central liquid. (B) FDG-PET/CT, status before treatment, showing three abdominal MTT manifestations: Right lobe of the liver $(26 \times 16 \times 17 \mathrm{~cm}$, SUV 10.8$)$, right lower quadrant of the abdomen next to the ileocecum $(3 \times 3.5 \mathrm{~cm}, \mathrm{SUV} 8.7)$, cranial to the urinary bladder $(1.7 \times 2.8 \mathrm{~cm}, \mathrm{SUV}$ 6.5). (C) FDG-PET/CT, status after neoadjuvant chemotherapy, surgery and adjuvant chemotherapy showing no residual tumor.

$1200 \mathrm{mg} / \mathrm{m}^{2}, 1 \mathrm{~h}$ intravenously for 1-5 days every 22 days. A second PET/CT-scan followed which demonstrated partial remission (WHO-criteria). The diameter of the hepatic mass had been reduced to $9 \times 6.5 \mathrm{~cm}$ (SUV 3.4); the two smaller manifestations close to the iliocecum and unrinary bladder were no longer detectable. Subsequently, the patient underwent laparotomy. Intraoperative exploration showed a soft and homogenous liver without evidence of intrahepatic metastases. However, the tumor was detected dorsal to the liver, originating from the retroperitoneum and infiltrating the diaphragm, the right Gerota fascia and the back side of the liver. The mass extended to the right hepatic vein. Complete surgical resection was performed by hemihepatectomy until all margins were histologically negative for residual tumor tissue (R0). No further tumor manifestations were seen macroscopically during surgery. The tumor surface seemed to be nearly completely necrotic. Indeed, final histological examination revealed exclusively necrotic tissue without evidence of vital MTT cells.

The postoperative course was uneventful, without any wound healing complications. Because of the good preoperative response, the patient subsequently received three additional cycles of PEI in an adjuvant setting. A follow-up FDG-PET/CT scan demonstrated complete remission without evidence of residual vital tumor manifestations or metastases
(Fig. 2C). Acute hematologic morbidity was noticeable after each cycle of PEI, with grade 4 leukopenia requiring G-CSF (Granulocyte-Colony Stimulating Factor).

Unfortunately, the patient had an abdominal reoccurrence of MTT after 9 months of follow-up, diagnosed using FDG$\mathrm{PET} / \mathrm{CT}$. One tumor was now infiltrating the dome of the urine bladder, as well as part of the vagina and intestine $(14 \times 9 \mathrm{~cm}$, SUV 7.1). A second tumor had resurfaced within the liver $(5.6 \times 4.2 \mathrm{~cm}$, SUV 13.1) and another one was infiltrating the small intestine $(3 \times 1 \mathrm{~cm}$, SUV 6.8). Lacking further surgical options, we started chemotherapy using an I/A regimen with ifosfamide $5000 \mathrm{mg} / \mathrm{m}^{2}$ for $24 \mathrm{~h}$ and adriamycin $75 \mathrm{mg} / \mathrm{m}^{2}$ for $1 \mathrm{~h}, 1$ day every 22 days. After three cycles of chemotherapy, partial remission was achieved with tumor response in the small pelvis $(7 \times 4.9 \mathrm{~cm})$, liver $(3 \times 3 \mathrm{~cm})$ and small intestine $(1.2 \mathrm{~cm})$. However, after three more cycles, tumor progression was diagnosed in the small pelvis $(8 \times 10 \mathrm{~cm})$, tumor breaking through the liver capsule $(5.7 \times 6.6 \mathrm{~cm})$, as well as new pleural and intrapulmonal manifestations. Finally, the patient died from progressive disease 26 months after diagnosis.

Case 2. A 24-year-old man with known NF-1 was hospitalized with a painful mass on the left side of his neck. A small nodule had first been recognized by the patient about two years before 
which had then, over the past 6 months, rapidly increased in size. On clinical examination, the patient presented with a non-shiftable, hard swelling on the neck, with proximally accentuated paresis and muscle atrophy of the left arm affecting nerve radicals C5, C6 and C7. An MRI-scan of the neck and cervical spine showed a heterogeneous tumor (9x7x5 cm) affecting the left cervical nerve plexus and ranging to the neuroforamen, close to the vertebral artery. Because of suspected malignancy, a tumor extirpation was performed in our department for neurosurgery. The pathological diagnosis of MTT was confirmed by evidence of malignant schwannoma and rhabdomyoblasts. Diagnosis was underlined by immunohistochemical staining. Unfortunately, the surgical resection was incomplete, with positive histological margins (R1). Postoperative CT-scans of the neck, thorax and abdomen showed no further tumor manifestations. Re-surgery by left neck dissection was performed, this time with histologically tumorfree margins (R0). Local irradiation of the operated area with $66 \mathrm{~Gy}$ of ${ }^{60} \mathrm{Co}$ followed. Two months after radiotherapy, the patient again complained of an increasing mass on the left of his neck, with radiating pain in the arm. An MRI scan of the neck again showed vital tumor $(4 \times 3.2 \mathrm{~cm})$, now enclosing the left arteria vertebralis and partially infiltrating the cervical spine.

The patient was referred to our department because of inoperability. We performed a total body FDG-PET/CT-scan, which confirmed the local reoccurrence of the cervical tumor (4.5x3.5 cm, SUV 3.8), now with additional multiple bipulmonal metastases (up to $5.8 \mathrm{~cm}$ in diameter, max. SUV 11.9). Because of advanced disease, four cycles of PEI were applicated. Grade 4 leukopenia was noted after each cycle of PEI, requiring G-CSF. Besides grade 2-3 nausea and/or vomiting, no non-hematologic toxicity was noted which could be related to the chemotherapy. Clinically, the pain was rapidly diminishing. After the third cycle of chemotherapy, successful stem-cell collection was performed for potential high-dose chemotherapy. After the fourth cycle, a staging performed with a FDG-PET/CT-scan showed partial remission of the pulmonary nodules (up to $2.7 \mathrm{~cm}$, max. SUV 7.5). The cervical manifestation was still constant in size, enclosing the arteria, but showed signs of central necrosis in an MRI-scan. The concept of experimental high-dose chemotherapy was refused by the patient and we administered two more cycles of conventional PEI. After the sixth cycle, progressive pulmonal metastases were diagnosed, with ongoing stable disease of the cervical tumor. A first cycle of MAID, with mesna, doxorubicin 20, ifosfamide 2.5 and dacarbacine 300 $\mathrm{mg} / \mathrm{m}^{2}$, was therefore administered for 3 days every 22 days. Again, G-CSF was given due to grade 4 neutropenia. Three weeks later the patient presented with a progressive cervical tumor and pulmonal metastases, as well as newly diagnosed cerebral metastases. The patient died of progressive disease, under the best supportive care, 18 months after diagnosis.

\section{Discussion}

MTT was first described by Masson in 1932 (11). The rare tumor is named after the Triton salamander (genus of triturus), which is able to regenerate limbs consisting of muscle, bone and nerve tissue if the cut end of the sciatic nerve is implanted into the soft tissue of its back (12). In 1973, Woodruff and colleagues coined the term 'malignant triton tumor' and defined it, following histopathology criteria, to establish a diagnosis: i) The neoplasm is connected to peripheral nerves or occurs in patients with NF-1. ii) Most of the neoplasm consists of Schwann cells. iii) The neoplasm contains rhabdomyoblasts. Immunohistochemical evaluation helps to distinguish between the different MPNSTs and to confirm the diagnosis of MTT (10).

Common sites of occurrence are the head and neck, and the trunk and lower extremities $(13,14)$. Tumors arising in the context of NF-1 display a male predominance, young age and common presentation in the head and neck region, as was presented here in case 2. Although the number of published MTT case reports is very low, it appears that the natural history of MTT is more aggressive in patients with NF-1 (15). In contrast, patients without NF-1 are older, show a female predominance and, most often, tumor manifestations on the trunk, consistent with patient 1 of this report (13). The prognosis is favorable for the head and neck and upper and lower extremities, and worse for the buttocks, trunk and retroperitoneum. However, MTT is a highly malignant tumor with a poor outcome $(1,5)$. The prognosis is worse than that for overall malignant schwannomas; overall survival and diseasefree survival are significantly influenced by the patient's age, tumor localization and size, the extent of the surgery, and the quality of the margins.

Currently, available publications in the worldwide database on the origin and, especially, on the treatment of MTT are very limited. It is always difficult to speculate on treatment guidelines when dealing with an extremely rare disease. However, as with many STSs, wide excision of MTT followed by postoperative radiation is the standard treatment of choice. It is the general consensus that suboptimal cytoreduction provides no survival advantage to patients with MTT (1). In case of marginal or incomplete resection, as presented in case 2, a new, enlarged surgical resection should always be discussed before administration of any adjuvant treatment. Moreover, the complete resection of the tumor, with or without adjuvant therapy, tends to portend improved survival over incomplete resection with or without adjuvant therapy (14). Therefore, although the benefit of optimal cytoreduction for the survival of advanced-staged MTT patients is undeniable, the feasibility of its achievement may vary depending on the surgeon, the selected patient population and the aggress-iveness of the tumor. The value of cytotoxic drugs remains questionable, as published experiences are limited $(7,16,17)$. Modern dosing regimens with ifosfamide, doxorubicin or cisplatin, in mono- or combination-therapy, display no convincing level 1 evidence (7). Chemotherapy is usually reserved for palliation in advanced or metastatic MTT disease (14). However, there is evidence to suggest that patients who have responded to preoperative chemotherapy should be treated with postoperative chemotherapy as well (18).

As Pisters stated, we are left with the conundrum of how to make meaningful progress against a disease like STS (7). He claimed that trials have to be developed focused on individual tumor subtypes. New treatment approaches are desperately needed which, with more than 50 known sub- 
types of STS, is very difficult to achieve. Molecular characterization of miscellaneous STSs could potentially identify subgroups and lead to a more specific course of therapy. At present, studies with molecular targeted therapeutics - e.g. inhibitors against vascular endothelial growth factor receptor, RAF-proteins, mammalian target of rapamycin or modern chemotherapeutics such as liposomal anthracycline, ecteinascidine (ET-743) or oral trofosfamide are under investigation.

The purpose of this report is to contribute to the available literature on experiences with MTT, not only because of its extreme rarity, but also in order to present clinical experiences of neoadjuvant therapy with the help of PET/CT as a diagnostic tool. Disease-free survival in patient 1 was achieved for 9 months after neoadjuvant chemotherapy with PEI resulted in a good clinical response, as was R0-resection of the tumor without histological evidence of vital MTT cells remaining. Patient 2 initially received surgery twice, followed by postoperative high-grade radiation. Chemotherapy was initiated because of advanced disease with local inoperability and additional pulmonary metastases, but treatment response was only slight.

MRI has previously been described as the imaging modality of choice for STS evaluation (8). Experiences with PET-scans for patients with STS have not been published before, but they have been shown to potentially offer advantages in detecting soft tissue tumors, local recurrence and metastases $(19,20)$. Their sensitivity to primary sarcomas ranges from 74 to $100 \%$ in literature; higher in high-grade compared to low-grade tumors. Especially in patients with NF-1, who frequently have a multiplicity of tumor-suspected swellings, this diagnostic tool can help in differentiation. As presented in our cases, MTT manifestations demonstrate strong FDG uptake in PET/CT, which helped us reliably assess tumor response and plan multimodal therapeutic interventions.

In conclusion, neoadjuvant chemotherapy plays a potentially important role in a multimodal approach with a subset of patients having high-grade, metastatic and primarily unresectable MTT. Patients who have responded to preoperative chemotherapy may benefit from adjuvant chemotherapy in order to facilitate subsequent surgery and to eradicate micrometastatic lesions. Nevertheless, the issue of pre- and postsurgical chemotherapy in patients with STS, and especially with MTT, still remains unsettled. Trials, for example protocols from the European Organization for Research and Treatment of Cancer, are still ongoing and will hopefully address and clarify this issue (21). Additionally, $\mathrm{PET} / \mathrm{CT}$ may represent a valuable diagnostic tool in the multimodal management of advanced MTT.

\section{References}

1. Stasik CJ and Tawfik O: Malignant peripheral nerve sheath tumor with rhabdomyo-sarcomatous differentiation (malignant triton tumor). Arch Pathol Lab Med 130: 1878-1881, 2006.

2. Daimaru Y, Hashimoto H and Enjoji M: Malignant 'triton' tumors: a clinicopathologic and immunohistochemical study of nine cases. Hum Pathol 15: 768-778, 1984.
3. Matsunou H, Shimoda T, Kakimoto S, Yamashita H, Ishikawa E and Mukai M: Histopathologic and immunohistochemical study of malignant tumors of peripheral nerve sheath (malignant schwannoma). Cancer 56: 2269-2279, 1985.

4. McComb EN, McComb RD, De Boer JM, Neff JR and Bridge JA: Cytogenetic analysis of a malignant triton tumor and a malignant peripheral nerve sheath tumor and a review of the literature. Cancer Genet Cytogenet 91: 8-12, 1996.

5. Yakulis R, Manack L and Murphy AI Jr: Postradiation malignant triton tumor. A case report and review of the literature. Arch Pathol Lab Med 120: 541-548, 1996.

6. Aydin MD, Yildirim U, Gundogdu C, Dursun O, Uysal HH and Ozdikici M: Malignant peripheral nerve sheath tumor of the orbit: case report and literature review. Skull Base 14: 109-114, 2004.

7. Pisters PWT: Preoperative chemotherapy and split-course radiation therapy for patients with localized soft tissue sarcomas: home run, base hit, or strike out? J Clin Oncol 24: 549-551, 2006.

8. Nosaka S and Kao SC: MRI of malignant 'triton' tumor in a child. Clin Imaging 17: 53-55, 1993.

9. Chijiwa K, Uchida K and Tateyama S: Immunohistochemical evaluation of canine peripheral nerve sheath tumors and other soft tissue sarcomas. Vet Pathol 41: 307-318, 2004.

10. Woodruff JM, Chernik NL, Smith MC, Millett WB and Foote FW Jr: Peripheral nerve tumors with rhabdomyosarcomatous differentiation (malignant 'Triton' tumors). Cancer 32: 426-439, 1973.

11. Masson P: Recklinghausen's neurofibromatosis, sensory neuromas and motor neuromas. Libman Anniversary Volume 2. The International Press, New York, pp793-802, 1932.

12. Locatelli P: Formation of membres surnumeraries. CR Assoc Anatomistes, 20e Reunion Turin. pp279-282, 1925.

13. Brooks JS, Freeman M and Enterline HT: Malignant triton tumors. Natural history and immunohistochemistry of nine new cases with literature review. Cancer 55: 2543-2549, 1985.

14. Ducatman BS, Scheithauer BW, Piepgras DG, Reiman HM and Ilstrup DM: Malignant peripheral nerve sheath tumor. A clinicopathologic study of 120 cases. Cancer 57: 2006-2021, 1986.

15. Aldlyami E, Dramis A, Grimer RJ, Abudu A, Carter SR and Tillman RM: Malignant triton tumor of the thigh - a retrospective analysis of nine cases. Eur J Surg Oncol 32: 808-810, 2006.

16. Kim ST, Kim CW, Han GC, Park C, Jang IH, Cha HE, Choi G and Lee HM: Malignant triton tumor of the nasal cavity. Head Neck 23: 1075-1078, 2001.

17. Kraybill WG, Harris J, Spiro IJ, Ettinger DS, De Laney TF, Blum RH, Lucas DR, Harmon DC, Letson GD and Eisenberg B: Phase II Study of neoadjuvant chemotherapy and radiation therapy in the management of high-risk, high-grade, soft tissue sarcomas of the extremities and body wall: Radiation Therapy Oncology Group Trial 9514. J Clin Oncol 24: 619-625, 2006.

18. Pezzi CM, Pollock RE, Evans HL, Lorigan JG, Pezzi TA, Benjamin RS and Romsdahl MM: Preoperative chemotherapy for soft-tissue sarcomas of the extremities. Ann Surg 211: 476-481, 1990.

19. Hicks RJ, Toner GC and Choong P: Clinical application of molecular imaging in sarcoma evaluation. Cancer Imaging 5: 66-72, 2005.

20. Schuetze SM, Rubin BP, Vernon C, Hawkins DS, Bruckner JD, Conrad EU III and Eary JF: Use of positron emission tomography in localized extremity soft tissue sarcoma treated with neoadjuvant chemotherapy. Cancer 103: 339-348, 2005.

21. De Laney TF, Spiro IJ, Siut HD, Gebhardt MC, Hornicek FJ, Mankin HJ, Rosenberg AL, Rosenthal DI, Miryousefi F, Ancukiewicz M and Harmon DC: Neoadjuvant chemotherapy and radiotherapy for large extremity soft-tissue sarcomas. Int $\mathbf{J}$ Radiat Oncol Biol Phys 56: 1117-1127, 2003. 